\title{
Principali risultati conseguiti durante I' osservazione geo- dinamica, opportunamente estesa nel tempo, di grandi dighe di sbarramento, e loro giustificazioni teoriche
}

\author{
P. CALOI - M. C. SPADE:
}

Ricevuto il 20 Febbraio 1966

Riasungo. - Vengono esaninati, melle loro ginstificazioni teoriche. i risultati di sistentatiche osservazioni geodinamiche (eseguite con l'ausilio di vibrometri, sismografi e clinograffi). compiute jresso grandi dighe dell'E.N.ES.

Anche da quest'esane. scaturisce la grinde utilità - che spesso confina con la necessití — di tali osservazioni. Per fuanto si riferisce all'uso dej clinografi, un quindicennio di registrazioni ottenute presso la diga ai Val Gallina, permette di far rientrare il tragico evento del Vajont in $m$ fenoneno di portata secolitre sperialmente attivo nell'ultima era geologica ucll'orogenesi alpiua; e. che. in condizioni particolari, può condure (eome hat condotto) ad anomale sitmazioni locali.

Sumary. -- Are here examined -- in their theoretical explanations the results of systematic geodynamic olsservations (carried out by vibrometers, seismograjphs and clinographs), arhieved on E.N.EL. big Dams.

This investigation also, shows the great nisefulness - being, inderi, a necessity - of such olservations.

A period of fiftees years of clinograplice records on the Val Gallina's Dan, include the Vajont's tragic event into a secular range phenomenon, particularly active - - during the last geologic Era -- in the Alpine orogenesis who, in speeial conditions, may be the cause (just like it happened) of anomalous local situations.

1. - Diverse dighe del Veneto, e le relative rocce d'imposta, a partire dal 1949, furono sottoposte a controllo geodinamico. Particolarmente seguite, furono le dighe di Pieve di Cadore, del Lumiei e del Vajont. Per alcune di asse tale controllo è tuttora in atto.

Il controllo geodinamico si applica, essenzialmente, nel rilevamento delle caratteristiche fondamentali del campo klastico nella zona interessata dalla diga, a delle loro eventuali variazioni nel tempo. 
I principali elementi del vontrollo sono:

a) Rilevamento del modulo elastico della roccia e del calcestruzzo (modulo di Young) e sue variazioni nel tempo.

b) Rilevamento della verticale apparente e delle sue variazioni: per cause periodiche (altcrnarsi delle stagioni, insolazione e sua variazione nell'anno, ecc.); secolari (di origine tettonica o comunque geologica); accidentali, legate a deformazioni locali del mezzo, come conseguenza della variazione delle curatteristiche elastiche, ecc.

c) Rilevamento delle brusche rotture d'equilibrio del campo elastico, per microsismicità naturale o provocata.

I rilievi, di cui al punto a), possono essere eseguiti con vibrometri, completati dall'ausilio di geofoni; quelli di oui al punto b) con fotoclinograf, mentre le indagini contemplate nel punto o) sono riservate a speciali stazioni sismiche.

Di questi strumenti è stato detto, a più riprese, in precedenti pubblicazioni (1-3).

2. - La determinazione del modulo d'elastieità è stata fatta (e ciò avveniva jer la prima volta in Europa) rilevando contemporaneamente le velocità di propagazione delle onde longitudinali e trasversali del mezzo. Talvolta ci si è scrviti dello sole onde longitudinali. Anche su questo argomento si ì a lungo disensso in precedenti lavori ( $\left.{ }^{4}\right)$ e qui non intendiamo ripetre'ci. Dobbiamo solo aggiungere ohe una delle obiezioni, recentemente mosse a questo metodo la grotencei italiani (ehe, finalmente, dopo circa venti anni, si somo aceorti della sua esistenza), consiste nel rilevare che le formule relativa avrebbro valore solo per un mezzo omogeneo, isotropo. A rigore, cio risponde al vero. Va però immediatamente sottolineato che tutti i tentativi fatti per tener conto dell'anisotropia del mezzo, oltre a comportare rilevanti complicazioni analitiche, hanno condotto a risultati, l'ordine di grandezza dei quali non si discosta sensibilmente da quello relatjvo al caso dell'isotropia, le differenze rientrando largamente entro i limiti di crove. Del resto, ehi ha qualche cognizione di sismologia, sa ehe i valori dei tempi di tragitto relle onde longitudinali e trasversali, ottenuti su percorsi di migliaia e migliaia di chilometri nell'interno della Terra, con le formule valevoli per mezzi isotropi, coincidono con i valori osservati fino al decino di secondo.

Fatta questa precisazione, ricapitoliamo rapidamente ciò che è stato largamente esposto in relazioni, a suo tempo inviate alla SADE, e in jubblicazioni specifiche ( $\left.{ }^{4}\right)$. 
Il primo fatto messo in luse fu la netta dimimuzione della velocità delle onde elastiche, rilerata dopo lo sbancamento, nei confronti dei vaJori ottenuti prima dell'inizio dei lavori di scaro. Questo, in tutti i masi sperimentati. Poiché il fatto si traduceva in una sensibile diminnzione del modnlo elastico, indicavamo il fenomeno col tedrnine decalimento (*) del modulo elastiro $\left(^{5}\right)$.

Il fatto fu attribuito, nella sua parte preponderante, alla deconpressione conseguente al distacen di grosse bancate di roccia. Il decadimento però non si arrastava al lavorio determirato dallo sbaneamento. Una volta costruita la diga, specie nelle zone d'imposta, esso riprendeva e continuava per anni, sia pure gradualmente attenuandosi, tendendo asintoticamente verso valori costanti (Figg. 1-t di Pieve di C.).

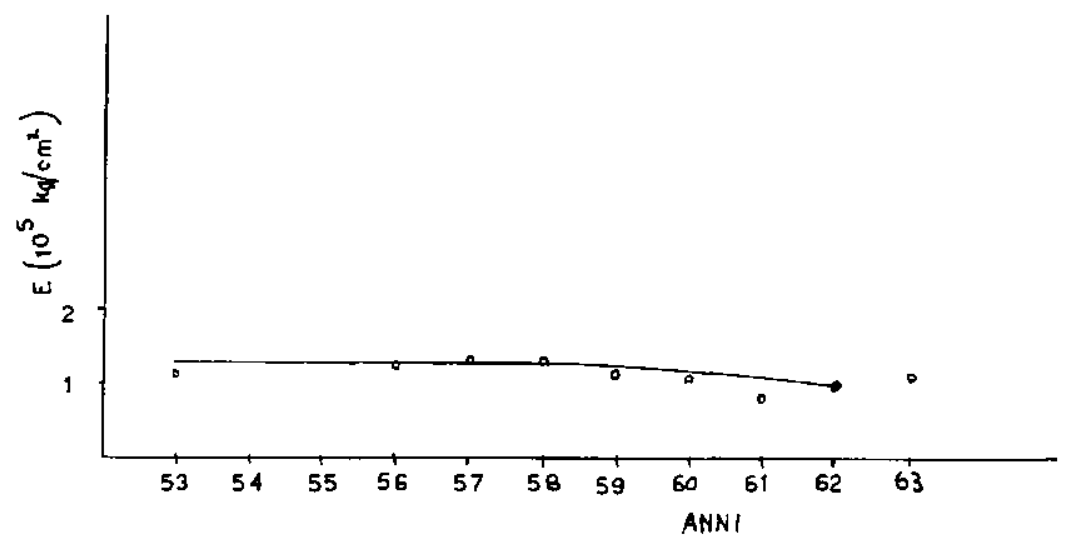

Fig. l - Diga di Pieve di Cadore. - Decadimento del modulo di elasticita per un tragitto in Spalla Destra.

Ciò fu spiegato $\left(^{5}\right)$ come consegruenza di mas minuta mierosismicità, provocata nel sistema roceioso a cui la dign è ancomata, dai continui movimenti eui lo sbarmanento viene sotoposto dib valiazioni di carieo nel bacino idrieo a monte e da valiazioni aella tomperatura esterna, per l'insolazione diurna e l'alternarsi delle stagioni.

La microsismiciti provocata fu infatiti messa in risalto dalla stazione sismica, funzionante presso la diga di Pieve di Cadore, a partire

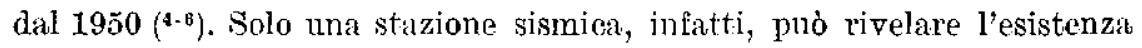
di una microsismicità locale, provocata o no. Per quanto concerne Pieve di Cadore, p. es., basterì osservare che delle parechie diecine di migliaia

(*) Il termine riusei ostico a greolorri e geotenici, portati addirittura a negare il fenomeno. Riteniamo the, attralmente, si siano ricreduti (almeno lo speriamo). 
di microscosse, registrate dalla stazione sismica nel primo quinquennio di funzionamento, solo un'infima parte - forse una diecina in tutto - fu avvertita dal personale addetto alla diga.

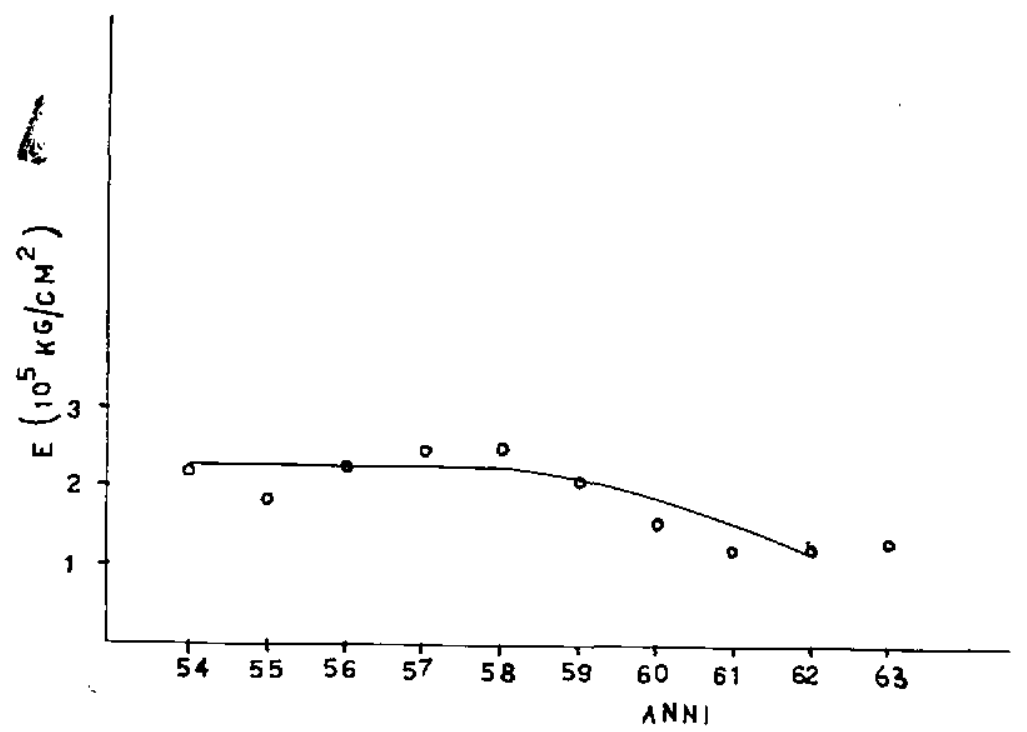

Fig. 2 - Diga di Pieve di Cadore. - Decadimento del modulo di elasticita in Spalla Sinistra per tragitti tracciati trasversalmente alla valle.

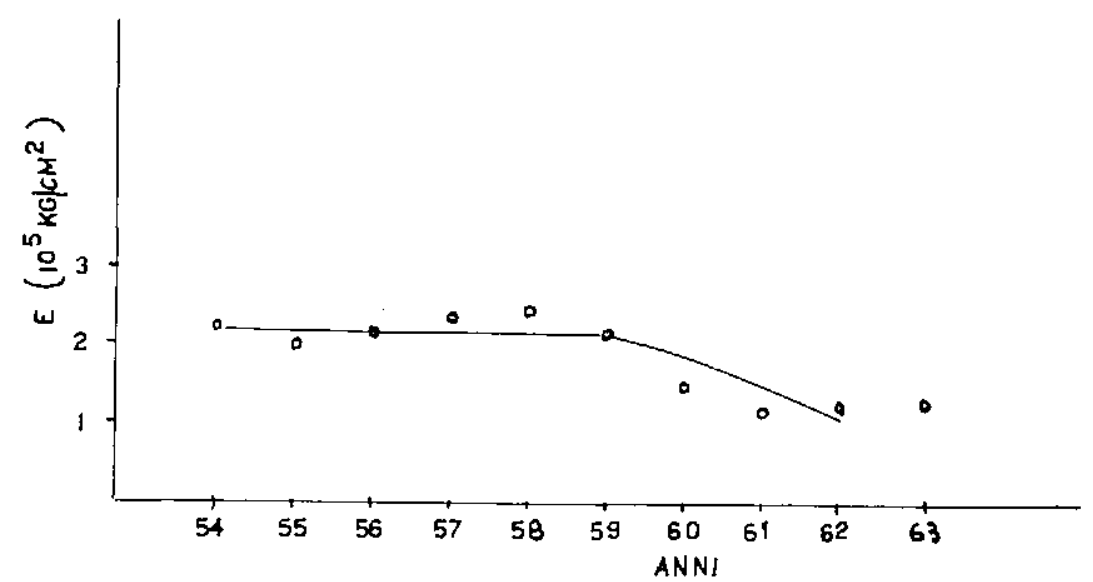

Fig. 3 - Diga di Pieve di Cadore. Decadimento del modulo di elasticità in Spalla Sinistra.

II deterioramento del campo elastico locale, si traduceva in una conseguente diminuzione della velocità delle onde elastiche, e quindi, nel decadimento del modulo elastico. 
Veniva spontaneo di attribuire tale deterioramento all'aumentatat porosità della roccia, come conseguenza del gran numero di piccole fratture, associate alle microscosse verificatesi nel mezzo $\left({ }^{6}\right)$.

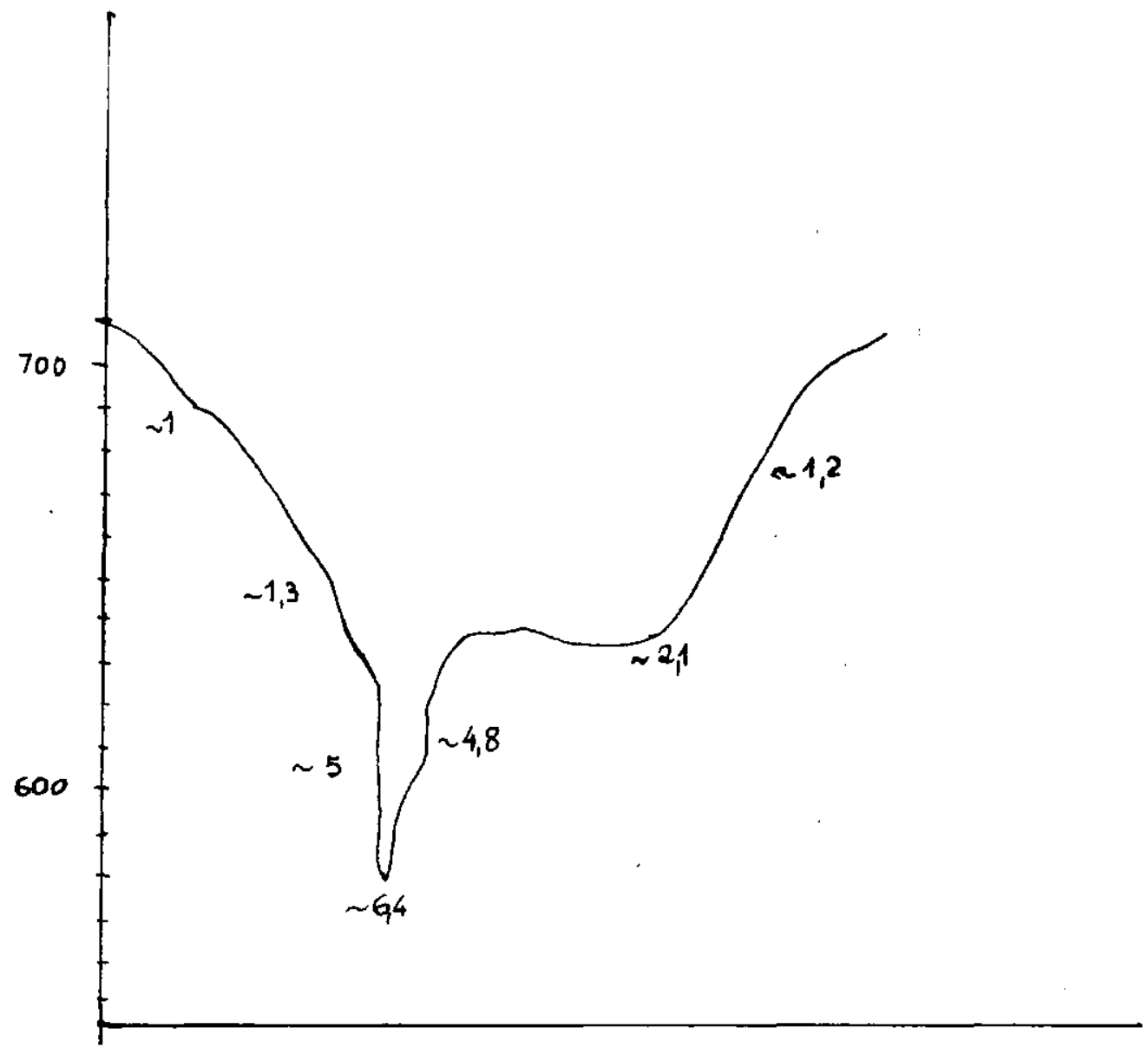

Fig. 4 - Diga di Pieve di Cadore. - Valori medi del modulo di elasticità nel 1962 per le vatie zone della strelta (lel Piave (in unità $10^{5} \mathrm{~kg} / \mathrm{cm}^{2}$ ).

3. - Accurate ricerche di laboratorio furono compiute sull'argonento della porosità e dei problemi connessi.

Lu porosità è indubbiamente uno dei più seri ostacoli nello studio dell'elasticità delle rocce. Una volta eliminato l'effetto della porosità con l'applicaziono di pressioni limite, molte caratteristiche delle rocce 
possono essere agevolmente studiate. L'esistenza di ruoti fra cristallo e cristallo costituisce pertanto un grosso ingombro nello studio delle proprietà fisiche delle rocce a pressione ordinaria.

Applicando elevato pressioni idlostatiche (più di 2000 atmosfere), l'effetto della porosità sulla velocità delle onde elastiche diviene piccolissimo, tanto da rendere superfluo il teneme eonto. Ad un iniziale aumento della pressione, la velocità cresee molto l'apidamenter, e solo per pressioni

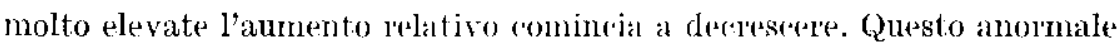
aumento della velocità alle basse pressioni, viene attribuito all'esistenza di vuoti fra i grani dellat roceia. Se la roccia è rompatta, l'iniziale aumento della velocità è più limitato.

Esiste, manifestamente, una l'elazione fra porosita e densità per rocee della stessa watura, nel senso (rhe l'una clesce quando l'altra diminuisce.

Furono fatte esperienze intese a dedure la velocita in funzione della densità di volume ("bulk clensity ") —e quindi della porosita in rocee dello stesso tipo. La densiti fu misurata per orni esemplare (campione di roccia), pesandolo e misurando l'immersione nel mer'urio.

It esperienze hanno dimostrato the, a pariti di altre condizioni, all'aumentare della porosità non solo diminuise'e la velocità ma anche l'ampiezza del movimento, a testimoniale che la porosità provoca anche un aumento di dissipaziorse. Is Figr. 5 dà la velocità delle onde longitudinali in funzione della, densità di volume per arenarie.

Per la determinazione dilla porosità rispetto alla densità di volume, esiste una formula empirica of tenuta da D. H. Davis ( ${ }^{7}$ ) da 601 campioni di arenarie ed altre rocce:

$$
\Phi=\left(1-\varrho_{B} / 2.65\right) \times 100
$$

dove

$$
\Phi=\text { porosità, } \varrho_{B}=\text { densità di volumr. }
$$

Nella relazione scritta, 2.65 rappresenta la media densità dei urani di minerale componenti: arenarie, argille, ecc. La porosità viene dedotta dalla densità di volume, sulla base della formula [1]. La relazione fra la velocità delle onde longitudinali e la porosità è riportata nella Fig. 6 . La velocità decresce esponenzialmente con la porosità, e la sua relazione è approssimativamente espressa dalla formula

$$
v_{P}=v_{P_{o}} e^{-\gamma \Phi}, \quad v_{P_{o}}=5750 \mathrm{~m} / \mathrm{sec}, \quad \gamma=0.036
$$


dove $v_{P_{0}}$ esprime la velocità delle onde longitudinali rell'esemplare di roccia, per il quale la porosità è nulla.

Dalla combinazione delle [1], [2] si tr'ue, per la velocità delle onde longitudinali in "arenarie " di nota densità di volume ("bulk density "):

$$
v_{P}(\mathrm{~m} / \mathrm{sec})=5750 \exp \left(1,36 \varrho_{B}-3.6\right)
$$

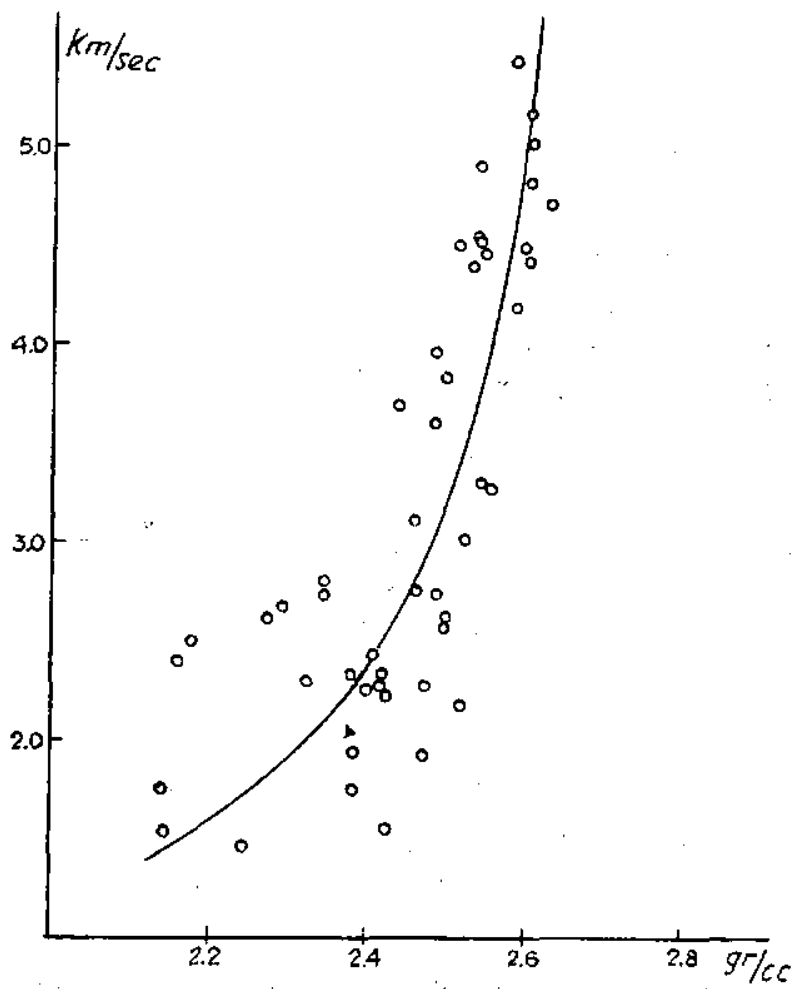

Fig. 5 - Velocità delle onde longitudinali in funzione della densità per arenarie (secondo D. Shimozuru).

Esistono altre relazioni sull'andamento della velocità con la porosità, specie in relazione alla profondità del materiale roccioso investigato $\left.{ }^{20}\right)$.

La teoria più rigorosa svolta su questo argomento resta, in ogni modo, quella sviluppata da. Y. Sato ${ }^{(B)}$. 
Per la velocità delle onde elastiche, ehe si propagano in materiale elastico contenente cavità sferiche vuote, egli ha dedotto le seguenti formule:

$$
\left\{\begin{array}{l}
v_{P}=v_{P_{o}}\left[1-(1-\varrho)\left\{\frac{1-1+8 \sigma_{o}}{6-5}+\frac{1-2 \sigma_{o}}{7-5 \sigma_{o}}\right\}\right], \\
v_{S}=v_{S_{o}}\left[1-(1-\varrho) \frac{4-5 \sigma_{o}}{7-5 \sigma_{o}}\right]
\end{array}\right.
$$

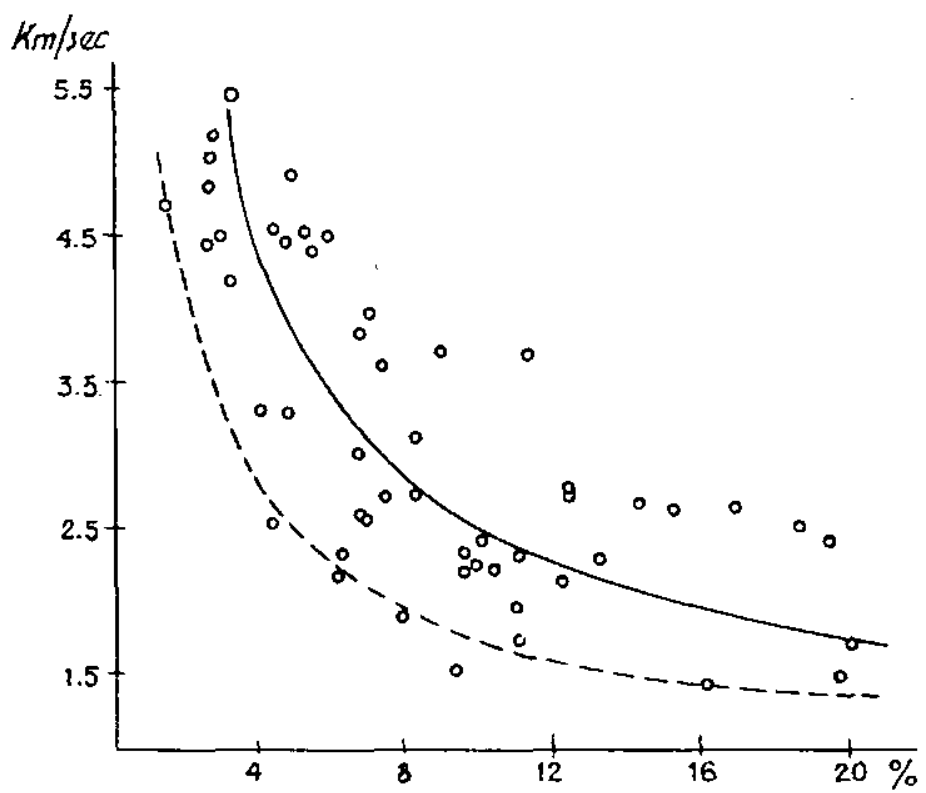

Fig. 6 - Velocità delle onde longitudinali in funzione della porosità per aremarie (secondo D. H. Davis).

dove $v_{P_{0}}, v_{S_{o}}$ rappresentano le velocità delle onde longitudinali e trasversali nel materiale privo di cavità,

$\varrho$, la densità relativa del materiale all'esame,

$\sigma_{o}$, il rapporto di Poisson per il materiale reale (privo di cavità), 1 - $\varrho$, la porosità.

$$
\begin{aligned}
& \text { Per } \sigma_{o}=\frac{1}{4}, \\
& v_{P}=v_{P_{0}}[1-0.768(1-\varrho)], \quad v_{S}=v_{S_{o}}[1-0.478(1-\varrho)] .
\end{aligned}
$$


La Fig. $T$ si riferisec alla tabulazione dei valori della velocità delle onde longitudinali in relazione con la porosità, calcolata da Sato.

Poiché si conoscono le velocità delle onde longitudinali (e trasversali) ottenute prima della costruzione delle dighe (di Pieve di Cadore, p. es., Vajont, Ambiesta, Val Gallina, ece) l'uso delle formule [1] - [2] - [3], oppure [4] e [5̃] permette di calcolare - in via approssimativa, naturalmente - l'aumento della porosità, nelle zone d'imposta, conseguente allo sbancamento ed ai movimenti della diga.

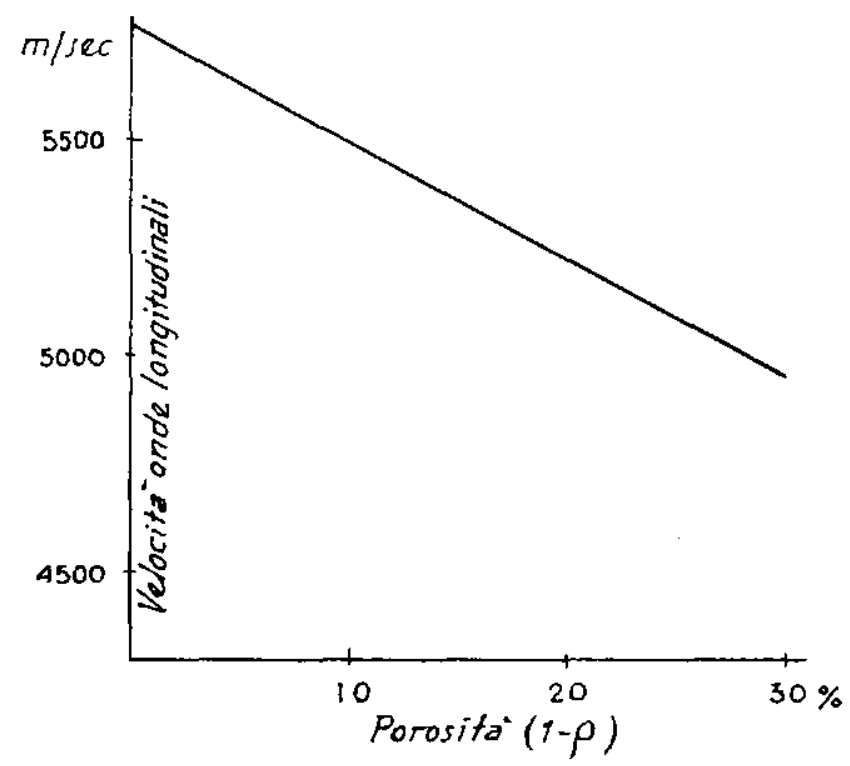

Fig. 7 - Andamento della velocità in funzione della porosita (secondo Y. Sato).

t. - Un altro fatto, messo ben presto in evidenza durante le indagini sul campo elastico nei pressi di una diga, fu la constatazione di velocità di propagazione delle onde elastiche, nella parte della roceia a contatto col bacino idrico, più o meno sensibilmente inferiori a quelle rilevate a valle dello sbarramento. Ciò si rese particolarmente manifesto nel bacino del Lumiei (Lago di Sauris).

Veniva spontaneo attribuire il fenomeno alla penetrazione dell'acqua nei pori della roceia. E tale fu appunto la spiegazione da noi data $\left(^{5}\right)$.

Anche su questo argomento sono state fatte, nel frattempo, indagini quantitative. 
Giova premettere alcune precisazioni cirea il significato delle pamole: contenuto d'acqua, porosita, rupporto di vuoto e rapporto di saturazione.

Si definisce come contenuto d'acqua $W$, il rapporto fia il suo peso $W_{w}$ e quello del materiale reale $W_{\varepsilon}$ :

$$
W=\frac{W_{w}}{W_{s}} \times 100
$$

Si definisce porosità $\Phi$ il rapporto del volume del vuoto $V_{n}$ a quello di tutto il sistema $r$ :

$$
\Phi=\frac{V_{n}}{V} \times 100=\left(1-\stackrel{\varrho_{B}}{\varrho}\right) \times 100
$$

dove $\varrho_{B}$, $\varrho$ sono la densità di volume e la densità sostanziale del materiale effettivo, rispettivamente.

Diremo rapporto di vuoto $e$, il rapporto fra il volume di vuoto $V_{n}$ e quello del máteriali $V_{s}$ :

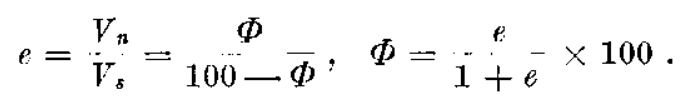

Infine diremo aliquota (grado) di saturazione $S$, il rapporto del volume di acqua $W_{w}$ a quello dell'intero vuoto $V_{n}$ :

$$
S=\frac{V_{w}}{V_{n}^{r}} \times 100
$$

Furono fatte indagini sulla variazione delle velocità delle onde elastiche con la siturazione di acqua.

E risultato che, in effetti, la velocitì delle onde elastiche viene fortemente influenzata dal grado di satmazione di acqua negli interstizi vuoti. La teoria più rigroresa svolta a questo riguardo è sempre quella di Y. Sato $\left({ }^{8}\right)$, che conduce alle seguenti espressioni, per quanto concerne le velocità di propagazione:

$$
\begin{aligned}
& v_{p}=v_{P_{o}}\left\{1-(1-\varrho) \frac{1}{2}\left[\frac{(1-k)\left(1+\sigma_{o}\right)}{2\left(1-2 \sigma_{o}\right)+k\left(1+\sigma_{o}\right)}+10 \frac{1-2 \sigma_{o}}{7-5 \sigma_{o}}-(1-D)\right]\right\}, \\
& v_{S}=v_{S_{o}}\left\{1-(1-\varrho) \frac{1}{2}\left[15 \frac{1-\sigma_{o}}{7-5 \sigma_{o}}-(1-D)\right]\right\},
\end{aligned}
$$


dove $\mathfrak{i}$ simboli hanno il significato già esposto; inoltre:

$k$ è il rapporto del "bulk modulus" del liquido $k_{1}$ a quello del matrviale reale $k_{0}\left(k=\frac{k_{1}}{k_{\text {in }}}\right)$;

$D$ è il rapporto della densità del liquirlo $\partial_{i}$ a quella del miteriale reade $\partial_{o}\left(D=\frac{\partial_{1}}{\partial_{o}}\right)$.

La Fig. 8 mostra la variazione di velocità delle onde longitudinali dovuta alla porosità per arenaria secea e saturata, calcolata con li:

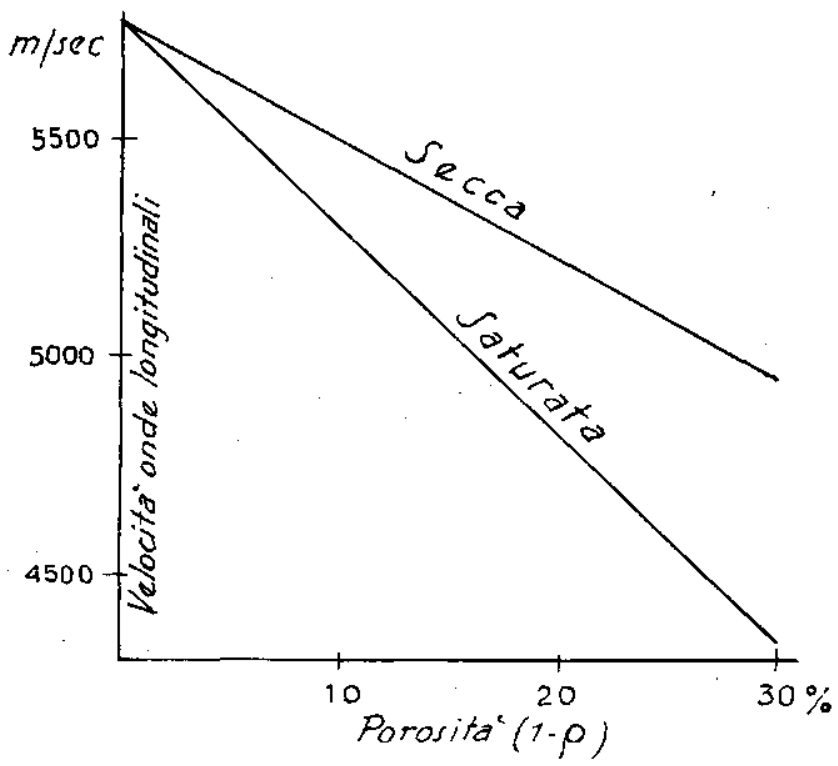

Fig. 8 - Velocita delle onde longitudinali in funzione della porositi per arenaria secea e satutrata (verondo Y. Sato).

formule [4] e [6]. La velocità prep arenuria saturata d'acqua è minore di quella par aremalia asciutta. La teoria, basata sul sistema di cavitì chiuse (cioè non intersomunicanti fra loro), non predice la velocità in funzione del grado di saturazione. In ogni modo, per esemplari non saturati, la velocità puó essere dedotta nella regione eompresa fra $\mathrm{i}$ due casi estremi, come in figur'a.

Le esperienze eseguite, sono genenclimente a favore della teoria di Sato. Frazer ( $\left.{ }^{\mathrm{I}}\right)$ trova che l'acqua interstiziale amenta l'effetto di angolarità. Per es., la porosità delle partionle di quarzo frantumato cresce 
dal $48 \%$ in un insieme asciut1o, al $54 \%$ in un insieme amido. Perciò, apparentemente l'acqua anmenta la porosità. In ciò deve vedersi la causa della diminuzione di velocità al crescere della saturazione.

In ogni caso, sembra che l'effetto sia decisamente in questo senso nei pressi della saturazione.

Furono fatte esperienze anche sulla variazione della velocitá in mezzi solidi con contenuto d'acqua (mescolanza di materiale solido e aequa), come sedimenti di oceano profondo, fondo di laghi, ecc.

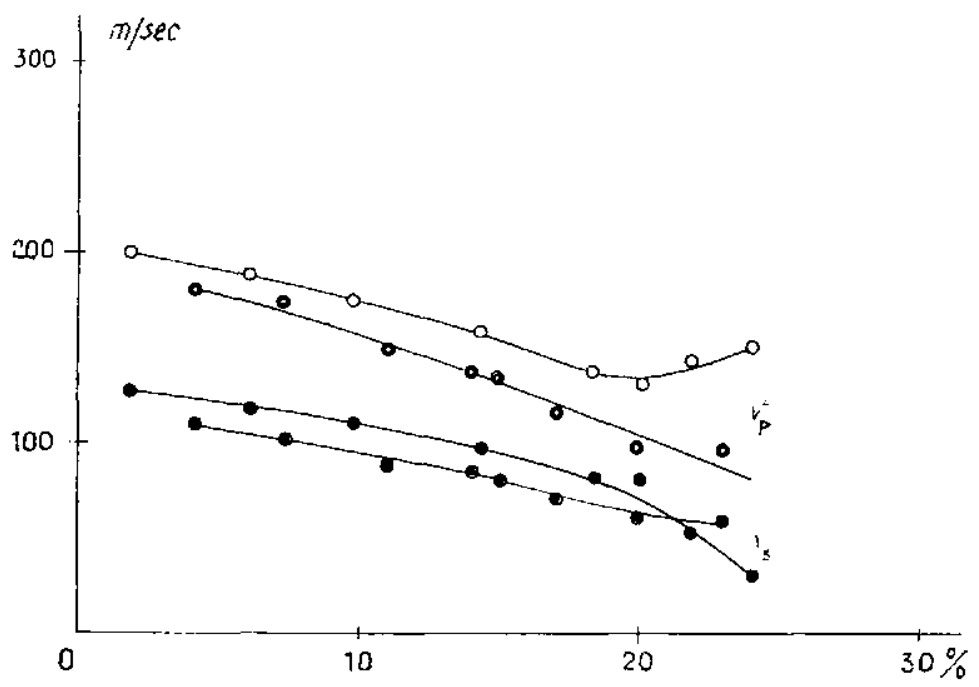

Fig. 9 - Velocità delle onde lousgitudinali e trasversali in funzione del contenuto d'aequa (secondo $K$. Iida).

E noto the l'osservazione sismologica mostra quanto sia picela la velocità delle onde sismiche in terreno soffice, nei confronti di quella delle roce. Tale velocità è fortemente affetta dal rontenuto d'acqua. Ricerche di laboratorio di K. Iida, condotte su terreni di diverso contenuto d'acqua, provano che la velocità delle onde longitudinali decresce al crescere del contenuto d'acqua. Ciò che va sottolineato è che la velocità di un miscuglio di due componenti, p. es. particelle solide ed acqua, varia con il rapporto di composizione ed $\dot{e}$ sempre considerevolmente al $d i$ sotto delle velocità di entrumbi $i$ componenti. Poiché lá velocità delle onde longitudinali per solidi a cui le particelle appartengono può varímre da 4 a $6 \mathrm{~km} / \mathrm{sece}$ e quella dell'acqua è $1.5 \mathrm{~km} / \mathrm{sec}$, la differerza fra le velocità misurate (dell'ordine delle centinaia di $\mathrm{m} / \mathrm{sec}$ - vedi 
Fig. 9) e quelle dei componenti is molto sylande. Lat velocità sembra raggiungere un minimo con un certo contenuto d'ac'qua, ce tende a laggiungere i 1500 miseo quando il comtenuto d'arequa tende al $100^{\circ}$ o, a il sistema risulta eomposto escelusivamente d'alequit.

5. - Da quanto precede, risulta chiaro comet, a paritì di porosità, possano esserc rexistrate velocità di propagazione sensibilmente diverse, in rocee della stessa natura, a valle c a monte di una diga (come è stato verifieato per le dighe in (amia e in (athore). Son solo, ma come possit spiegarsi perehè nello stesso massiecio rorrioso - senza che si sia verificata variazione apprezzabile nella sua porosita - si possano ottenere, nel tempo, valori della velocità sopta o sotfo la media: ciò può dipendere dal diverso grato di saturazione in cui la rocela è venuta a trovarsi in epoche diverse; come ì stato eflettivamente constatato nella zona d'imposta della diga di Pieve di Cadore, in spondat destra e in sponda sinistra (Figgr. 1-3).

Un altro fatto ò degno di menzione: Jinché l'ossatura solida di un mezzo conserva "arattere di continuita, la relocita delle onde elastiche condotte dal mezzo è sempre elevata, quantunque la sua porosita - in senso lato - possa provocarne un'apprezzabile diminuzione. Non appena il tessuto solido comincia ar incrinassi, la velociti delle onde elastiche diminuisee bruscamente; jer ridursi a valori bassissimi in caso di frattmazione multipla, cle renda il mezzo una s moplice sovrapposizione di elementi, singolamente companti, ma senzal reciproco legame. In questo senso, l'osservazione continta la lampo alastico nelle zone d'imposta

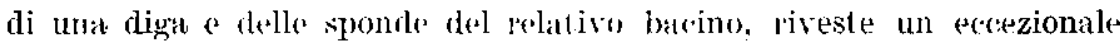
interesses, rome sensibilissinia misura di rontrollo. Ena testimonianza preziosa, a questo riguardo, è stata datal dal romportamento, in campo elastico, dell'ammasso roceioso sovmatunta in sinistra la disa di Pontesei: nell'intervallo di porhi anni (dal 1952 al 1958), la velocita delle onde longitudinali del romplesiso roeriuso or detto, serese da $3500 \mathrm{~m} / \mathrm{sec}$ eat a

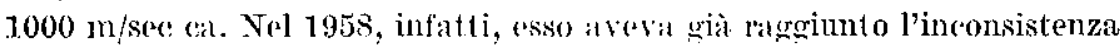
del materiale di frana; c solo un tempestivo inter'vento di alleggerimento della zona valso a sconsiulure l'effettivo movimento franoso. Un'altra ben più drammatica proval a conlerma di queste conelusioni è stata offerta dallo sperone roccioso in sponda sinistra a monte della diga del Vajont, risultato ancora compatto nel Dicembre del 1959 e che un anno dopo - nel Dicembre del 1960 - si era vivelato elasticamente debellato; conseguenza dell'azione di demolizione, deteminata nel frattempo, dalle centinaia di microscosse verificatesi nella zona $\left({ }^{17}\right)$. 
6. - Anisotropia elcstica delle rocee. Un altro problema di complessa trattazione è quello lexato all'anisotropia delle rocee.

F noto the roces dello stesso tipo possono presentare considerevoli differenze di velocità, indipendentemente dal loro stato di porosità. Per es., le velocità delle onde longitudinali nel marmo possono variare da $2.8 \mathrm{~km} / \mathrm{sec}$ a $7.3 \mathrm{~km} / \mathrm{sece}$. Fra le cause di tali variazioni, una delle più efficenti può essere l'anisotropia, o, romunqur', le deviazioni dalla perfetta isotropia delle roces.

In natura l'anisotropia elastifar delle roces ignes a metamortiohe è diversa da quella delle rocesedimentarie. Roces ignee ordinarie, quali il granito, l'andesite, la diorite, apparentemente appaiono isotropiche. In effetti, anche in questi tipi di rocce, il metodo ad ultrasuoni può rivelare variazioni di veloeità delle onde longitudinali e trasversali a seconda della. direzione. In ogni caso, si tratta di anisotjopia poco alecentuata. Essa viene attribuita alla natura eterogenea delle rocee, Irgata sopra tutto alla orientazione dei cristalli componenti. Se essi sono distribuiti a caso, in tutte le direzioni possibili, allowa gli aggregati policristallini risultano elasticamente isotrofici; in tal mso, verramo a mancare variazioni direzionali di velocità.

Nelle rocce sedimentiarie, tali variazioni direzionali di volocità possono essere accentuate. Ciò deriva dall meeanismo di sedimentazione o dal modo di associazione dei grani componenti.

Furono fatte ricerche sui due tipi di anisotropia. Noi accemeremo qui soltanto al seconclo tipo, quello the più ci interessa.

Il fenomeno dell'anisotropia rli velocità in rocere stratificate o sedimentarie è stato ripetutamerte osservato in rio'rehe di prospezione. sismich.

Il grado di anisotropia di relocilà si suole indiad'e con il cosi detto fattore di anisotropia $A$, definito come il apporto dellat velocità Iungo gli strati alla velocità considerata perpendicolumente agli strati. P. es., per sedimenti di carbonato, giacenti fra m 230 e m 2622 sotto la superficie, Uhrig ( $\left.{ }^{12}\right)$ trova un fattore $A$ rariabile fra 1,10 e 1,19. Ma il divario può essere anche maggiore. Così Nisu ed Omote $\left({ }^{13}\right)$ sperimentando sopra una rupe di arenaria trotarono un fattore di anisotropia pari a 1,83 (Fig. 10). L'anisotropia di velocità per le roce sedimentarie deriva da due diversi meceanismi. Uno è legato alla loro struttura laminata o periodica, l'altro alla struttura dell'associazione ("packing") dei grani componenti. Il primo è quello dell'urgilla (stratifienta), il secondo quello dell'arenaria.

Furono fatte misure di laboratorio con il metodo degli ultrasuoni. Un campione di arenaria, prelevata ad una profondità di $70 \mathrm{~m}$ e sago- 
mato, fu esaminato secondo due direzioni ortogonali ed una intermedia: come mostra la figma e la tay. di Fig. 11. La velocità massima, lungo una direzione $(X)$ risulta minima nella direzione ortogonale $(Z)$.

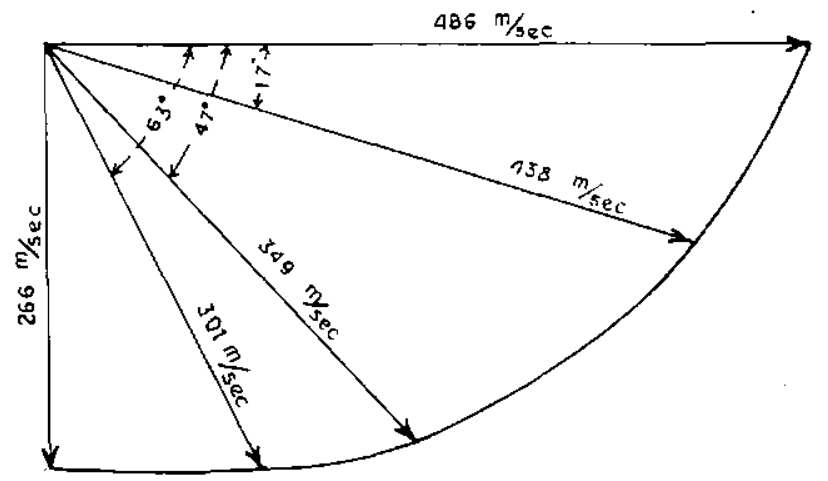

Fig. 10 - Anisotropia dellil velociti in un blocco di arenaria (secoutlo X. Nasu e G. Omote).

Anche la trasmissione dell'energia è diversa, a seconda delle direzioni: essa infatti viene fortemente attenuata nella direzione perpendicolare $(X)$ all'asse verticale $(Z)$, dove si osserva il minimo di velocità. In questo raso il fattore di anisotropia risultò di $1 . \bar{t}$.

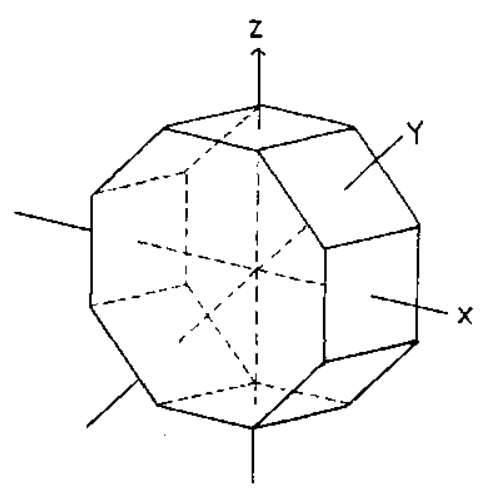

\begin{tabular}{|cl|}
\hline Dirzzione & Velocita \\
\hline$Z$ & $1210 \mathrm{~m} / \mathrm{sec}$ \\
$Y$ & $1560 \quad "$ \\
$X$ & $2098 \quad "$ \\
\hline
\end{tabular}

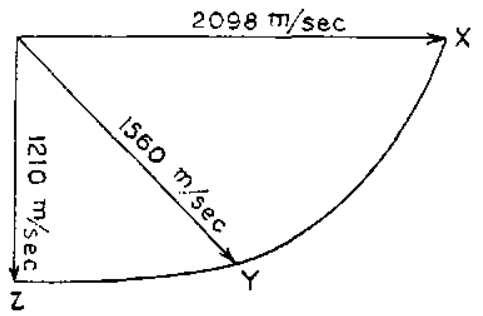

Fig. 11 - Anisotropia della velocità in un esemplare di arenariat (tratto da una profondità di $70 \mathrm{~m}$ ) di non chiara anisotropia strutturale (secondo D. shimozuru). 
Ir natura, con particolare riguardo alle roces d'imposta di una diga, lungro la sezione di una valle, le stratificazioni appaiono generalmente più o meno inclinate, lungo giaciture spesso contorte, comunque fortemente piexate. Le determinazioni della valocita conducono pertanto nella genteralità dei casi - a valori intermedi.

Pal arele nozioni sulle variazioni di velocità - e quindi del nodulo elastico - nel fempo, si sperimenta orviamente su direzioni fisse, prestabilite.

7. - Resterebbe da aceennale all'elasticità delle roce sotto pressioni limiti; nla di essa avemmo modo di ocenuarei a più riprese (4), $\theta$ qui riteniamo superfluo ripetereri.

8. - Sull'uso dei clinografi presso $i$ grandi sbarramenti. - Sull'utilità dei clinografi nel rilevamento delle condizioni di stabilità locale (esistenza o meno di bloceli confinenti, animati da moti relativi diversi, ecc.), sul rikatanento delle valiazioni della verticaje apparente in senso lato, sull'insostituibila tunzione di controllo eontinuo, sensibile, preciso, immediato, is stato da noi ampiamente riferito in numerose relazioni e pubblicazioni.

Qui ei preme soffermeres su aleunj aspetti delle osservalzioni clinografiche, che remromo il elinografo un prepioso complemento del sismografo e del vibrometro, nei rilevamenti delle valiazioni del eampo elastieo. Può infatti rivelaure variazioni permanenti della verticale apparente, in relazione con variazioni permanenti delle caratteristiche elastiche della roceia, come is stato messo in rhiara evideraza dalle registrazioni clinografiche nolla roretia d'imposta, in spalla destra, della diga di Pieve di Cadore $\left({ }^{6}\right)$.

Il suo wo contimuato nell'intermo di una digat, a comineiare dall'ultimazione dello sbartanento, olt le a rivelare i moti lenti o rapidi, periodici e non periodiei, sistemutici a aecidentali deI manufatto, fornisce dati evidenti sulla variazione nel tempo della risposta rlastica del calcestruzzo. Si nota, innanzi tutto, un graduale irrigidimento del calcestruzzo nei primi anni della costruzione della diga (rivelato, fra l'altro, dall'attenuazione dellonda diurna alla base della Diga - Pieve di Cadore, Ambiesta, Val Gallina, ... - dal lento migrere in direzione spalla-spalla della risultante dei moti del pulvino o tampone, legati ad asimmetria d'insolazione, inizialmente con sensibile componente anche verso valle-monteAmbiestal, ... - . 


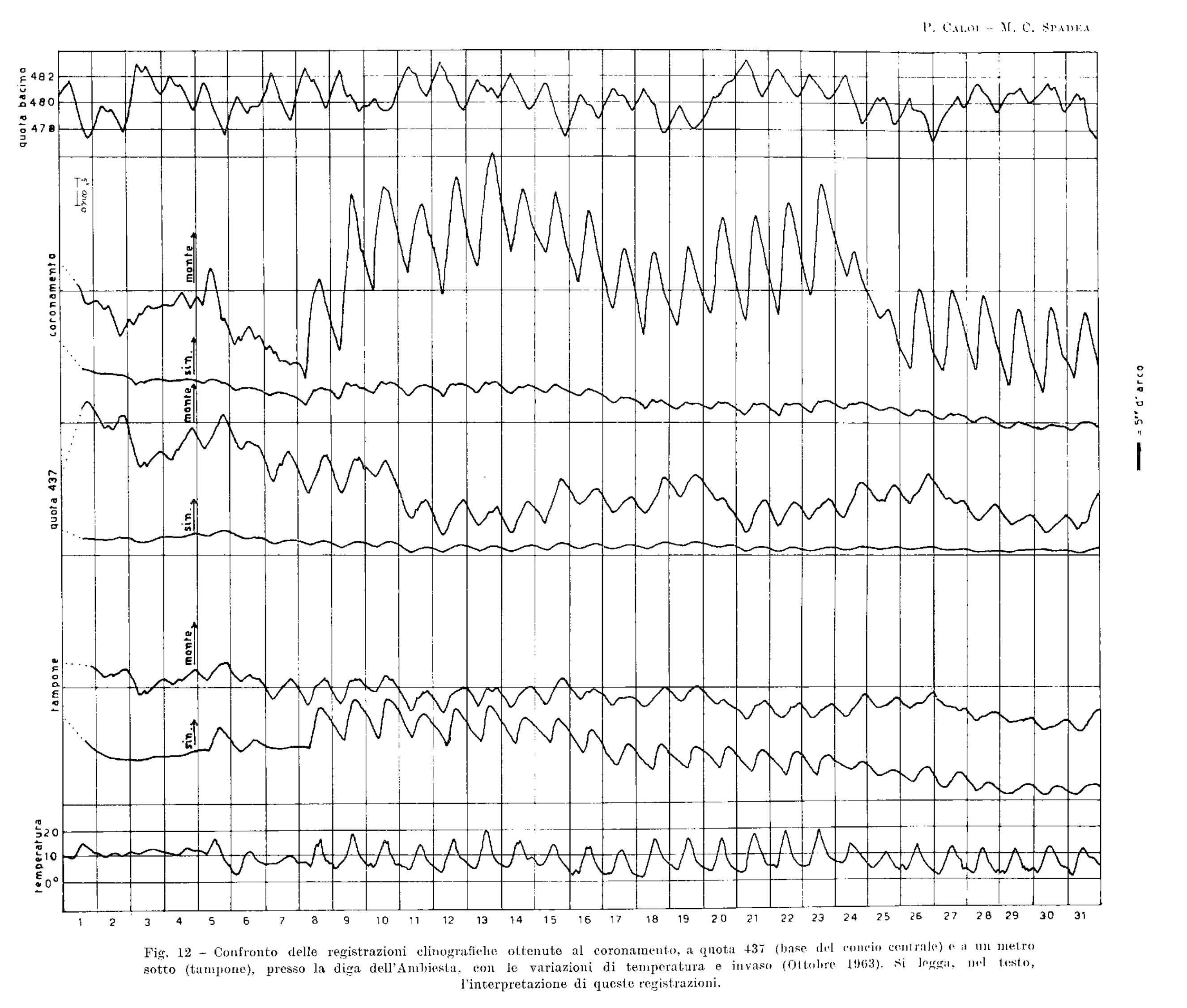




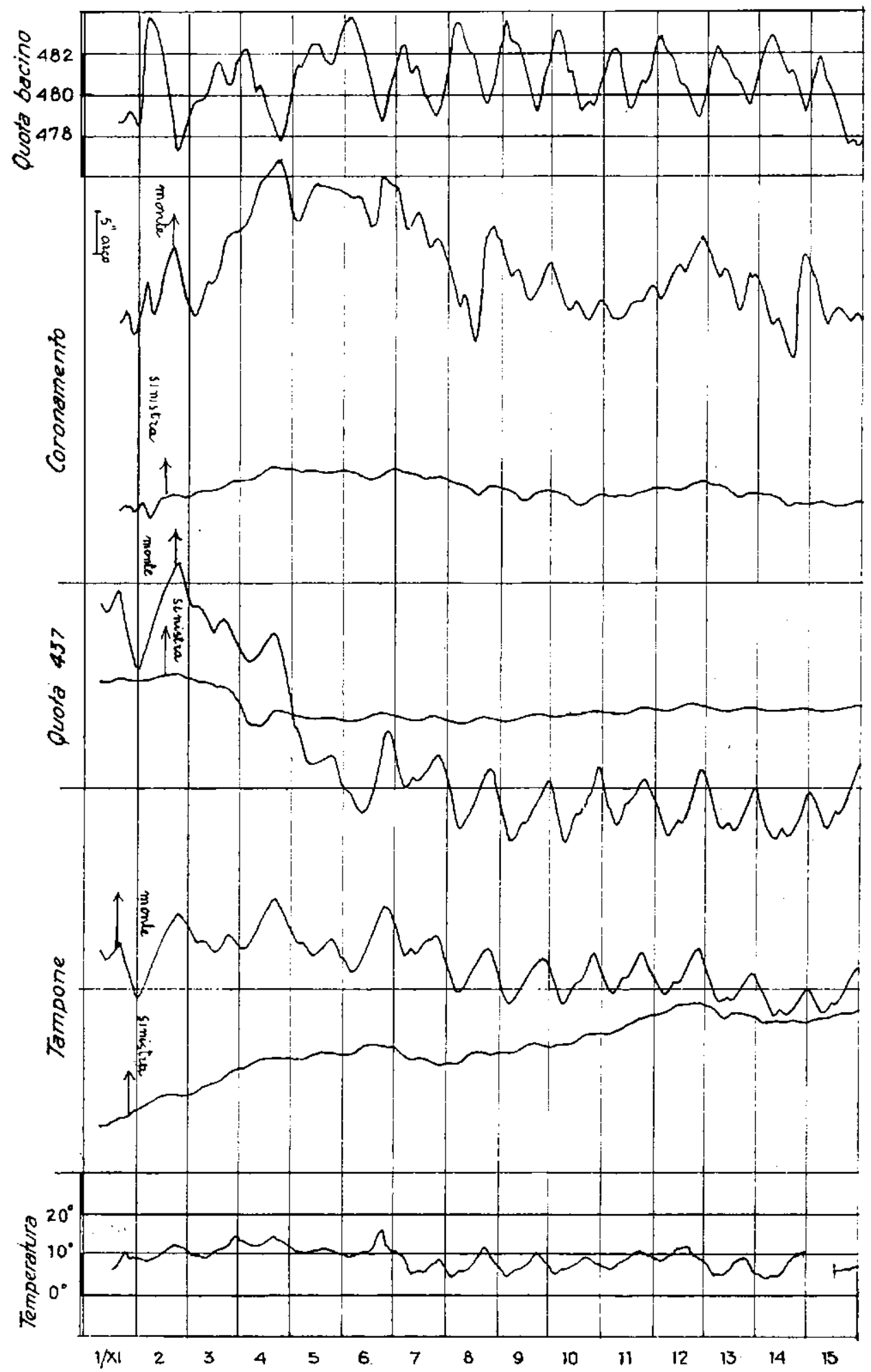

Fig. 13 - Confronto dati clinografici con dati temperatura-invaso, ottenuti nel Novembre 1963 presso le postazioni elinografiche site al coronamento, a quota $437 \mathrm{e}$, a un metro sotto nel tampone della diga dell'Ambiesta. 
L'osservazione elinegration prova poi che costruila per sopportare in modo uniforme le: pressioni provenienti dall'invaso, generalmente una diga si comporta come ligida nei confronti delle variazioni dell'invaso stesso (salvo casi di nna sorta di eflecto di "raldrizzamento nintermedio,

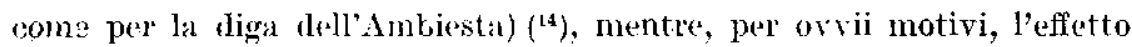

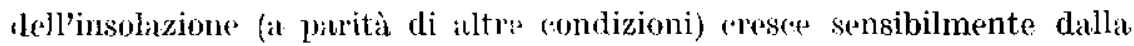
base al coronamento $\left({ }^{15}\right)$.

Di particolare interesse risulta - nelle rivelazioni dell'osservazione dinografica - il comportamento del pulvino presso alcune dithe nei confronti delle variazioni della tempreratura esterna dellaria, e, in particolare, dell'insolazione. Nel aso di asimmetria di insolazione (abbastanza comune nel amplesso delle dighe) il pulvino integra; per cosi dire, gli effetti dellazione dinamial legata all'insolazione stessa, nel senso che subisce l'inetruale distribuzione delle tensioni verticali nei singoli conci, come conseruenzat del diverso riscaldamento degrli stessi. Arviene così che, mentre alla base del concio centrale londa diurna non influisce in modo apprezzabile sulla variazione della verticule apparente (specialmente in direzione spalla-spalla), un motro sotto, il clinografo associato al pulvino, registra ampie onde diume, quasi esclusivamente nella direzione spalla-spalla. Tale affet to es particolarmente netto nella diga dell'Ambiesta $\left({ }^{14}\right)$; un po" meno areent uato presso la diga di Pontesei.

Un'altra caratteristica dinamica r.he distingue il pulvino dai ronei soviastanti è la seguente. In tatte le dighe le variazioni dell'invaso si trasferiseono in netie variazioni della verticale apparente fino alla base dei conei. E naturale ehe, sui conci centralli, le sa dette variazioni abbiano luogo, quasi esclusivamente, in direzione valle-monte. Nelle dighe, come in quella dell'Ambiesta, dove alla base - separate da un solo metro di quota - funzionano sulla stessa verticale due identiche coppie di clinoirati una ancorata al roncio eentrale, laltra al sottostante pulvino o tampone, le registrazioni delle variazioni di pressione da parte dellinvaso vengono registiate identiehe nella forma (anche nei minimi particolari), però lievemente diverse nell'ampiezza; una lexgera diminuzione osservandosi in quelle oltenute sul pulvino (tampone). Il che sta a significare che la superficie di discontinuità dinamical esistente fra il pulvino o tampone e il concio sovrastante, è sufficiente a provocare ana leggera attenuazione dei movimenti (analoga alla dissipazione che subiscono le onde elastiche nellattraversamento di una superficie di discontinuità). Tale fonomeno appare chiaramente registrato presso la diga dell'Ambiesta (Figg. 12, 13). I fatti acennati non devono stupire: essi rientrano nell'ambito dei micromovinenti. 

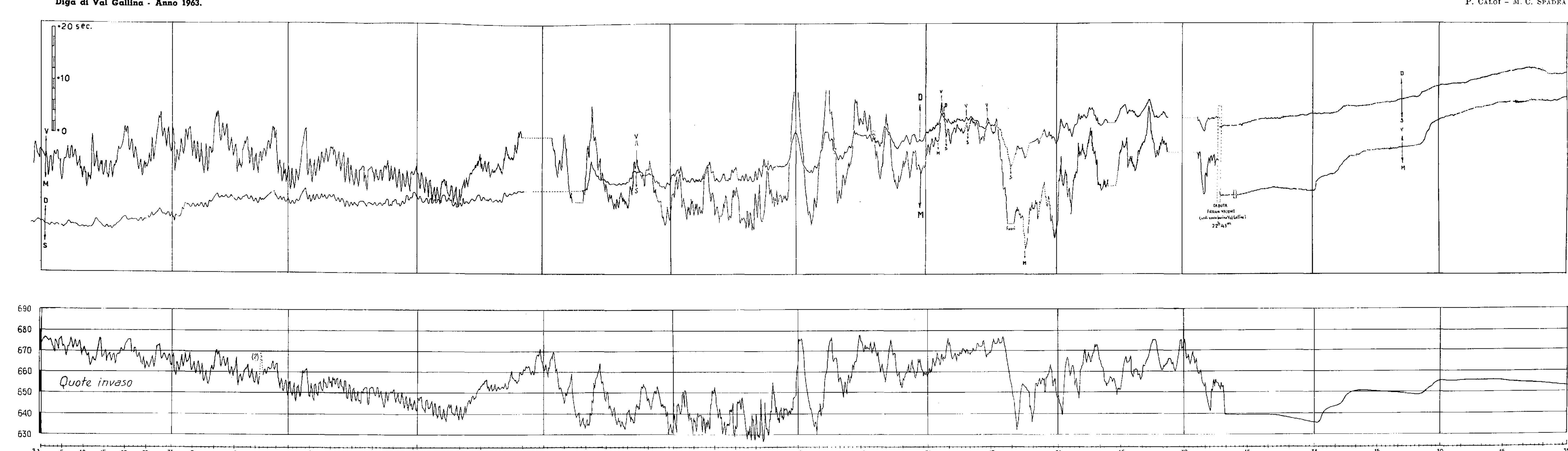

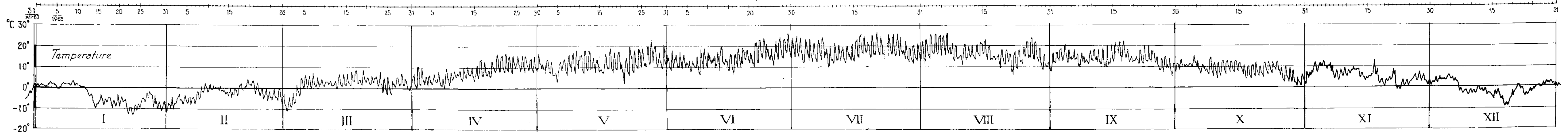



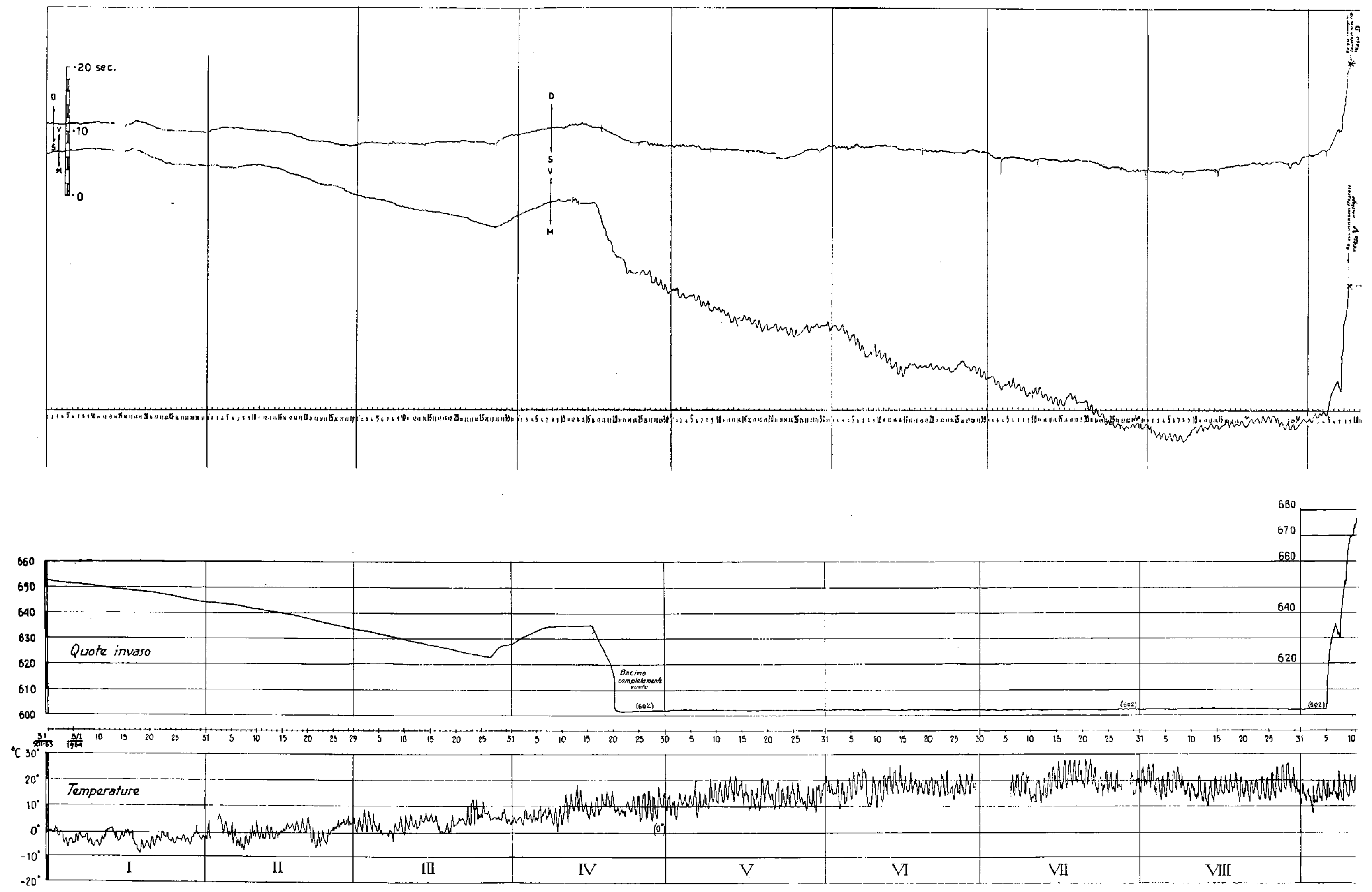

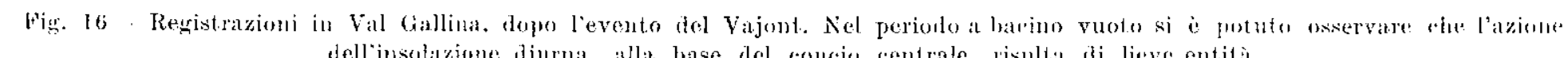

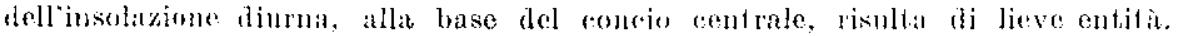


Mal anche altri fatti possono assere rivelati dat fotoclinografi dopo un sufficientemente prohumgato fimzionamento presso una diga: alcuni sistematici, altri leyati all'ambiente gesologico, in palticolane tettonico. Fra i primi, merita particolare menzione il lento, progressivo flettere verso valle del corpo della diga, spereie nella sua parte centrale (fenomeno espresso, fra i tenici, con la brutta locuzione di "effetto pancia "). A questo riguardo, possiamo citare il chiaro nsmpio fornito dalla diga di Val Gallina, alla base del eui concio rentrale funziona, dal 1951, un fotoelinografo a due componenti, una orientata secondo la direzione vallemonte, l'altra nella direzione a $90^{\circ}$ (spalla destra-spalla sinistra).

Cone vedremo più partitamente fral poco, presso questa diga le variazioni normali di (arien - derivanti dalle variazioni di livello delle acque nel bacino idrio — si traducono in variazioni della vertideale apparente lungo una linea formante con l'asse della diga un angolo di $12^{\circ}-15^{\circ}$; nel senso che unil spinta verso valle provoca un moto angolare risultante nel quildante valle-clestra, nentre un alleggerimento del carieo determinat una rotazione ne] quadrante monte-sinistra. Ebbene, da quando è in funzione il fotoclinograto, è stata registrata una costante "deriva" della verticale apparente vorso valle, a eui si soveappongono naturalmente molti altri movimenti, legati a diverse cause. Tale deriva, nella sua quasi totalita, e senz'altro da attribuire all'effetto, sopra aceennato, di flessione verso valle dello sbarramento. Nol periodo 1951-1965, la "aduta : verso valle della parte centrale desla diga è stata di ia 284 sec: 11 areo (Fig. 1.t).

Acenniamo ora anli alt ri movimenti. Sut1o lazione dell'invaso, come si e detto, le variazioni angolari si verificano lungo una linea che forma con l'asse della diga un angolo di $13^{\circ}-14^{\circ}$ cirea (Fimg. 15, 16). L'ouda diuma non è apprezzabile alli base della digu. Ivi infatiti il clinografo registra fedelmente le minime variazioni dellinvaso, dhe risultano in rapporto di perfetta proporzionaliti con le variazioni della verticales apparente $\left({ }^{2}, 3,4\right)$. La poor rilevanza dellonda diuma alla base della diga è confermata dai periodi di registruzione "s bacino vuoto: essa ̀̀ effettiva nei mesi axtumo-inverno (Fig. 16), m(ntl' appare appena aceennata nei mesi più calli (Figr. 17). La variazione della temperatura dell'aria, come presso le altre dighe, fal ruotare la diga a monte per temperatura crescente, a valle per temperatura decrescente. Ciò si rende più manifesto a barino vuoto. Questo, in condizioni normali. In certi periodi però, si verificano movimenti che non possono essere spiegati con nessuna delle cause sopera citate. Vedasi la Fig. 14, che riassume, mediandole, le osservazioni empiute dad 1951 al 1964. 
Solo cause naturali, ambientali, intese in senso lato, possono essere invocate per dar lagione di simili movimenti; in particolare, cause legate alla tettonica della zona. Come già è stato provato altrove $\left({ }^{16}\right)$, queste possono essere chiaramente rivelate dai clinografi. Nal caso specifico, quella

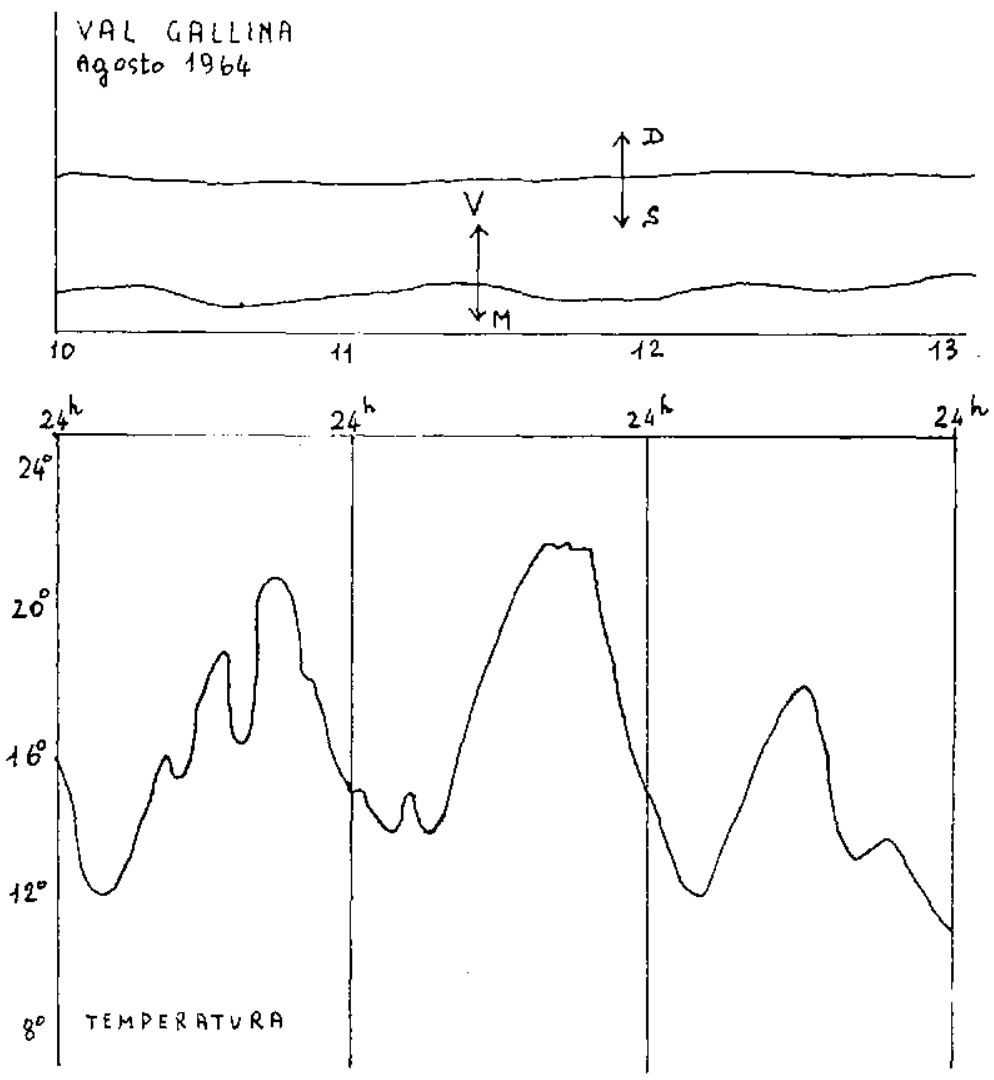

Fig. 17 - Un esempio di confronto fra dati clinografici e dati di temperatura ed invaso ottenuti presso la postazione di Val Gallina, a bacino vuoto.

$$
5 \mathrm{~mm}=1^{\prime \prime} \text {. }
$$

specie di grande cappio che la verticale apparente è stata obbligata a seguire dal 15-VII-1952 al 26-IV-1953 (v. Fig. 14), lisulta probabilmente dal contrasto di movimenti cui sono ancora soggette le stratificazioni esterne della Terra, specialmente nelle zone dove l'orogenesi ha potentemente agito nolle ultime ere geologiche $\left({ }^{17}\right)$. Il comporsi di moti, pure se generalmente unidirezionali ma localmente di diversa ampiezza, o addirittura di ampiezza variabile anche nello stasso luogo, non possono non tradursi in 
una sorta di moto risultante torsionale, di rui il capplo citato puó essere portato a testimonianza. In certi periodi, il contrasto fra i nassice i ehe formano la valle può essere tale da costringrere la diga decisamente in una rotazione in direzione forzata, soverchiante i suoi normali movimenti. Ciò che si è palesemente rerificato nel periodo dal 26-IV-1953 al 26-V-1954, in cuj lo sbarramento effettuó una sensibile, esclusiva pressione monte-valle, come costrettovi da un azione di "schiacriamento" fra le due sponde.

Ma dove la deviazione dai movimenti legati alla temperatura e all'invaso si è manifestata, nel periodo arcennato, più accentuata è nella quasi costante "deriva" verso la sponda destra della valle. Ad intervalli di arresto ne sono seguiti altri in cui il fenomeno appar' notevolmente accelerato. Vedasi ancora la Fir. 1.t. L'ultima fase del fenomeno riveste, al riguardo, particolare interesse: quella che prende le mosse dal Maggio 1960. Da quell'epoca, con lablentamenti e accelerazioni, la deriva verso destra ha carattere pressoché rontinuativo. A questo punto, va ricordato che lia Val Gallina si trova immediatamente a Sud della valle del Vajont (entrambi i torrenti sono tributari del Piave), dalla quale appare divisa dal massiccio del Toe. Non sembra azzardato collegare il fenomeno in questione a quanto stava maturando sulla sinistra del Fajont, alle pendiei del Toe. Risale infatti al 22 Magrio 1960 l'inizio della microsismicità nella stretta del Vajont, a monte della diga, in sponda sinistra $\left({ }^{17}\right)$, microsismieità ehe, alternando periodi (li intensificata frequenza ad altri di relativa calma, cloveva in poco piǹ di tre anmi demolire l'edificio roccioso che reggeva la sovlastante zona di frana. Non sembra, ripetiamo, azzardato associare la sistematica flessione detla diga di Val Gallina verso Nord, specialmente nel periodo sopra aceennato, ad un contrasto tetitonico interessante l'intera zona compresia fra le due valli contigue, dal Grep Nudo al Toc; contristo tettonico che aveva nella forra del Vajont il suo punto più eritico, a dove doreva sfociare nell'immane scoseendimento del 9 ottobre 1963.

Il fatto che la flessione verso la spalla destra della diga di Val Gallina sia continuata (Fig. 18) anche dopo il tragico evento del Vajont, sembra testimoniare a favore di questa ipotesi. Quell'evento, e il perdurare di questo fenomeno, sarebbero due aspetti diversi di uno stesso grandioso movimento tettonico, interessante la zona nella sua totalità $e$ non soltanto in superficie.

Negli ultimi anri, è un pullulare all'estero di studi sui moti recenti della crosta terrestre e sulle loro possibili caluse. Non è quj, naturalmente, il luogo per soffermarei a lungo su questi studi. Riteniamo però interes- 
sante osservare che, tali movimenti, vengono attribuiti a dislocazioni sub-crostali o a residui di moti convetitivi, ritenuti liberamente agenti in epoche geologiehe passate, ed ai quali ogroi si è portati ad attribuire i massimi corrugramenti della superficie terestre $\left({ }^{18-21}\right)$. Frat tutti, merita

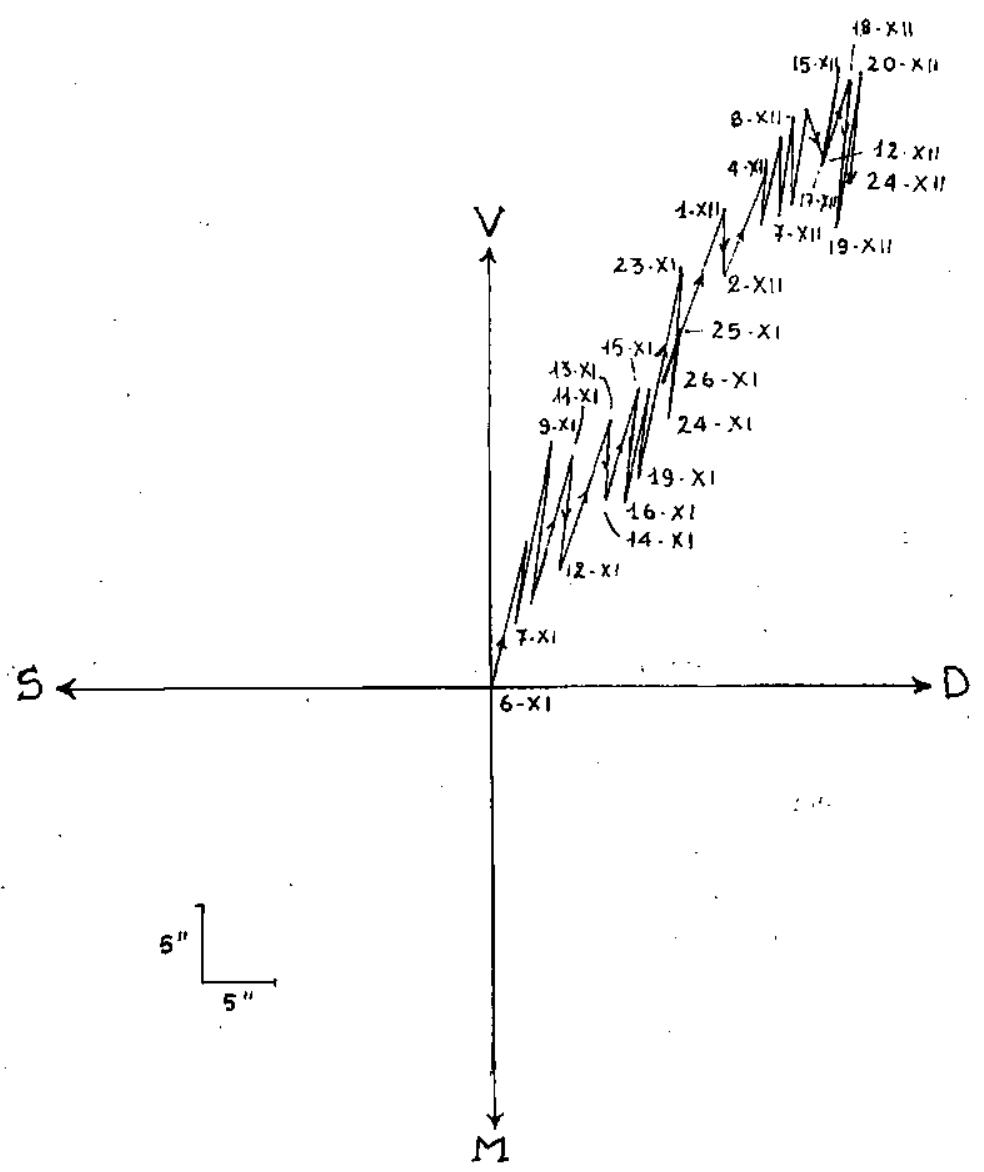

Fig. 18 - Risultante movimenti augolari subito dopo l'evento del Vajont.

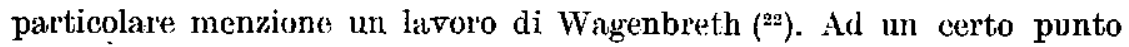
del suo lavoro, accettando le conclusioni di Pflue $\left({ }^{23}\right)$, egli ritiene che nell'Europa centrale le spinte (tensioni), ancora in atto, nella crosta terrestre siano come da Fig. 19.

L'esistenza di faglie o di discontinuità o comunque di inomogeneità attraverso la crosta terrestre (comunissime in regioni montuose), può 
generare situazioni locali del tipo di quella rappresentata nella Fig. 20. Colpisce l'analogia con quanto si è andato osservando attraverso gli anni nella zona Val Gallina-Valle del Vajont.

Tenuto conto della direzione della spinta subcrostale (Fig. 19), la deriva verso la spalla destra in Val Gallina e i fenomeni sismici osservati nella stretta del Vajont appaiono legati ad un'unica causa. Nelle nostre rolazioni

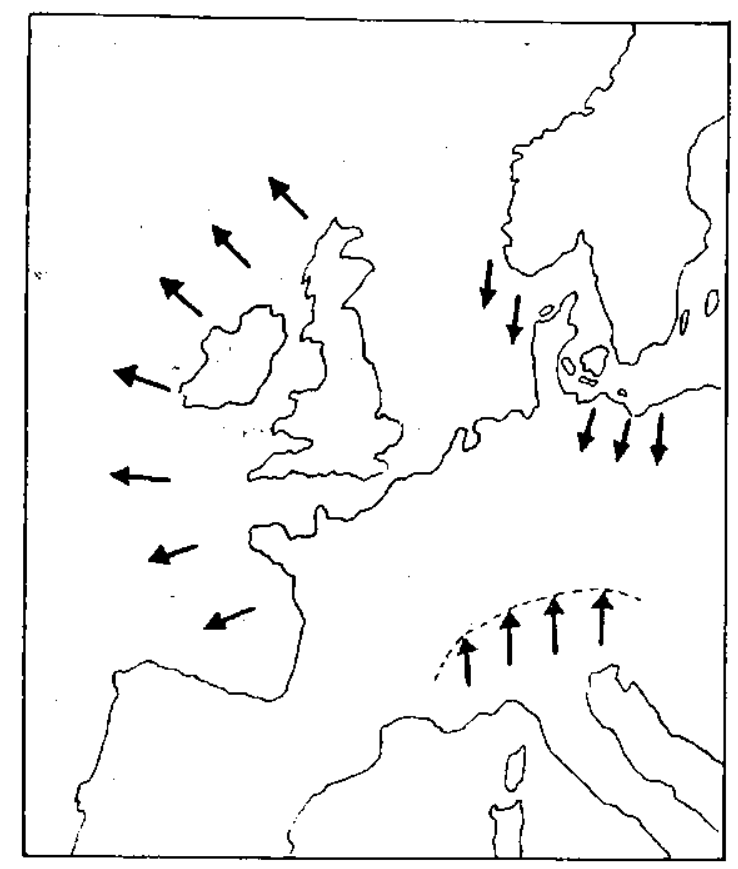

Fig. 19 -- Orientamentu di pressioni alpidiche, legate a correnti subcrostali (secondo H. D. Pflug).

alla SADE del 1962-1963, sottolineavamo l'esistenza di un contrasto tettonico in sponda sinistra del Vajont, a monte della diga $\left({ }^{17}\right)$, contrasto che si manifestava sotto yli aspetti di uno sprofondamento immediatamente a sinistra del torrente e di un innalzamento a ventaglio lungo le pendici del Toe. Quest'ultimo fenomeno era da noi attribuito ad un residuo di orogenesi $\left({ }^{17}\right)$. Collegando quelle nostre conclusioni al meccanismo suggerito dalla trattazione di Wagenbreth, si resta sorpresi nel constatare come tale meccanismo possa in pieno spirgare quanto è arvenuto nella stretta: del Vajont: si noti infatti che le dilatazioni vengono a corrispondere alla piccola frecicia che indica moto verso il basso (Fig. 20), mentre le com- 
pressioni osservate dalla stazione sismica hanno la loro esatita rispondenza nella piceola freecia associata al sollevamento. In tal modo, verrobbe pure confermato il modello ad origine singola delle microseosse $\left({ }^{1 i}\right)$.

Le registrazioni clinografiche ottenute presso la diga di Val Gallina testimonierebbero al favore di questo meccanismo. Cio consentirebbe di concludere che l'evento del Vajont era legato ad un contrasto tettonico di vaste proporzioni, probabilmente non ancor's conchiuso. Va infatti
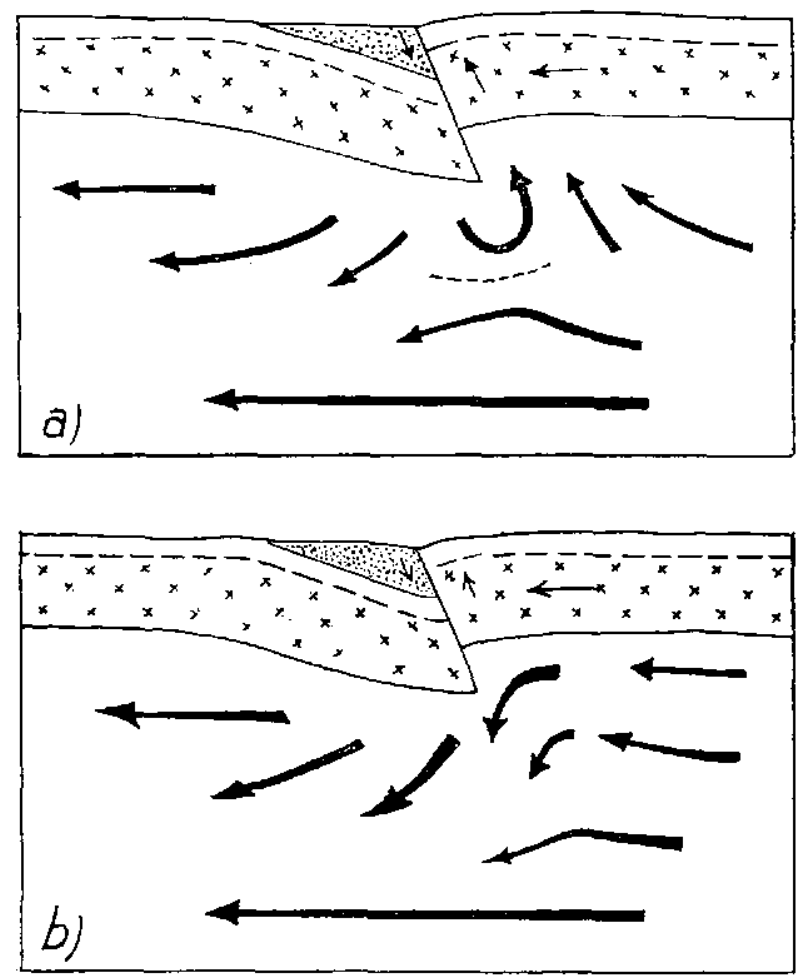

Wig. 20 - Anomalie locali determinate da ostacoli alle correnti suberostali. Grosse frece: direzioni della rorrente subcrostale (secondo Wagenbreth).

osservato, a questo riguardo, che - sulla base delle registrazioni clinografiche di Val Gallina - la deriva verso destra ebbe una fase di arresto nei mesi che precedettero il tragico 9 Ottobre 1963. Sembra quasi che, in questa fase, la spinta verso Nord della parte esterna della crosta avesse trovato un intoppo al conseguente slitiamento della parte in lentissimo moto. Dopo l'enorme scoscendimento del 9 Ottobre, la de- 
riva verso destra denunciata dai clinografi di Val Gallina è lentamente ripresa, come se in quella tremenda notte del 9 Ottobre l'intoppo su detto avesse ceduto.

\section{BIBLIOGRAFIA}

(1) Calor l'., Il pendolo orizzontale come clinometro. "Ann. Geofis. ", III (1950).

$\left({ }^{2}\right)$ Calor P., Ossercamioni sismiche e elinografiche presso grandi dighe di sbarramento. "Ann. Geofis.", VI (1953).

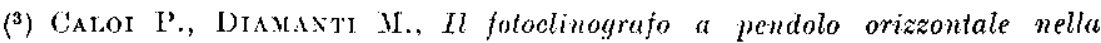
teoria e nell applicasione. "L'Energia Flettrica", XXXIII (1956).

(4) Calor P., Aspetti della dinamica di rocee calesstruzzo ed acque. "Anm. Geofis." XV (1\$62). (Contiene, fra l'altro, un'ampia bibliografia sui lavori pubblicati sullargomento dall'Autore, a partire dal 1950).

(5) Calor P., SPAUEA II. (., Decadimento del modulo elastico in roccia a contatto con basini idrici artificiali. "Ann. Geofis." VI (1954).

(') Cator P'., La geodinamien al sernizio delle grandi dighe. "Ann. Geofis. ". XVI (1963).

(7) Davis D). II., "Joml. (reol, ", 62, (1954).

(*) Sato Y., Telocity of Elastic Waves Propagated in Media with Small Holes. "Bull. Fartha Res. lnst. "T Tokvo, XXX (1952) e XXXI (1953).

(9) SATO Y., l. c., 185, (1952).

$\left({ }^{10}\right)$ Sirmozunu D., Elastirity of Rorks and Some Related Geophysical Problems. "Jap. Journal Geophys, ", 2, 3, (1960).

(11) F'razer H. Y., "Joltr. Gieol. ", 43, 910, (1935).

(12) LTHRig C. F., "Grophysies", 20, 774, (1955).

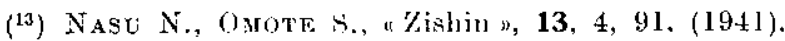

(14) Canor P., aspetti gerdinamici della diga dell Ambiesta, "Am. Geofis." XVII, (1964).

(15) Calor P., La geofisien e le grandi dighe: "Ii Eneroia Elettriea "XXXIX. (1962).

(16) Calor P., SPADEa II. (... Relazioni fra lente variazioni dinclinazione e moti sismici in zona ad elevata sismicità. "Rend. Ace. Naz. Lincei", XVIII, 3 (1955).

(17) Calor P., Lievento del J'ajont nei suoi aspetti geodinamiei. "Arm. Geofis.", XIX, (1966).

(18) SchempgGer A. E., Statistical thalysis of Reent Fault Plane Solutions of Earthquakrs. "Bull. Seism. Soc. Amer," 49 (1959). 
(19) ScheidegGer A. E., Grosstektonische Bedeulung von Erdbebenherdmechanismen. "Zeitschrift für Geophysik", 31, Heft 6, (1965).

$\left({ }^{20}\right)$ Lu. Paur II. and SCHeneggerr A. L., $4 n$ intensive local application of Lensen's $X_{\text {sallo }}$ stress Theory to the Sturgeon Lake south area of Alberta. "Bull. of Canadian Petroleum Geologry", 13, 3, (1965).

$\left.{ }^{21}\right\rangle$ DE Borr Jelle, Paleomagnetic Indications of Megatectonic Movements in the T'ethys. "Journ. of Geophys. Research ", 70, 4, (1965).

${ }^{(22)}$ WAGENBRETH O., Zur theoretisehen und regionalen Deutung des Elbtalgrabens und der Lausitzer Überschiebung. "Geologie", Jalırg. 15, Heft $1,(1966)$.

(23) PFLUG H. D., Versuch einer telktonischen Deutung der tertiären Sedimentationsräume NW-Deutschlands. "Freiberger Forsch-H., C. 80"(1960). 